Arcos Severo, M., Gutiérrez Cedillo, J. G., Balderas Plata, M. Á., \& Martínez García, C. G. (2021). Servicios ecosistémicos de provisión suministrados por agroecosistemas de huertos familiares del Estado de México. Revista de Biología Tropical, 69(3), 1069-1078. https://doi.org/10.15517/rbt. v69i3.46561

\title{
Servicios ecosistémicos de provisión suministrados por agroecosistemas de huertos familiares del Estado de México
}

\author{
Minerva Arcos Severo1; (D https://orcid.org/0000-0002-9728-0015 \\ Jesús Gastón Gutiérrez Cedillo²*; (D) https://orcid.org/0000-0002-0089-701X \\ Miguel Ángel Balderas Plata ${ }^{2}$; (D) https://orcid.org/0000-0003-2889-3923 \\ Carlos Galdino Martínez García ${ }^{3}$; (D) https://orcid.org/0000-0001-9924-3376
}

1. Facultad de Química, Universidad Autónoma del Estado de México, Paseo Colón, 50130, Toluca, Estado de México, México; min.arcos@hotmail.com

2. Facultad de Geografía, Universidad Autónoma del Estado de México, Cerro de Coatepec, s/n, Toluca, Estado de México, México; jggc1321@yahoo.com.mx (*Correspondencia),mplata@colpos.mx

3. Instituto de Ciencias Agropecuarias y Rurales, UniversidaAd Autónoma del Estado de México, Carretera TolucaIxtlahuaca Km 14.5, Toluca, Estado de México, México; cgmartinezg@uaemex.mx

Recibido 22-IV-2021. C Corregido 13-VIII-2021. Aceptado 09-IX-2021.

\begin{abstract}
Ecosystem provision services provided by agroecosystems of family gardens in the State of Mexico
\end{abstract}

Introduction: Provisioning ecosystem services (ES) provided by agroecosystems of family orchards (AEFO) refer to the tangible benefits that people obtain from the ecosystem. Although AEFO have been widely studied, few studies show their approach from an integral perspective, which incorporates biophysical and economic characteristics at the same time.

Objective: To carry out an integral analysis of provisioning ecosystem services provided by agroecosystems of family orchards from State of Mexico.

Methods: Information about provisioning ecosystem services (identification, uses, destination and income generation) were collected between March to December 2019 from three rural communities by involving 42 families with a family orchard using semi-structured interviews, questionnaires, species inventories, direct observation and field trips.

Results: A sum of six provisioning ecosystem services were identified, which were associated to 212 species, distributed in 172 genera and 82 families. The main uses that the owners of the orchards gave to provisioning ES were ornamental (51\%), followed by food (39\%) and medicinal (32\%), where species such as Cymbopogon citratus, Persea americana, Punica granatum, Zea mays e Inga jinicuil, had the highest use values (U. V. = 4). The destination of the production was self-consumption, sale and exchange, being the first the main survival strategy used by households. Sale of edible products in fresh, as well as their derivatives, represented an average gross income of $1333.47 \mathrm{USD} /$ year for the owners of the family orchards.

Conclusions: Provisioning ecosystem services provided by family orchards seen as an important source of resources, which allow the households of rural communities classified in extreme poverty to cover multiple needs.

Key words: agrobiodiversity; economic valuation; tropical agroecosystems; interdisciplinary analysis; local knowledge. 
Los servicios ecosistémicos (SE), se refieren a los beneficios que las personas obtienen de los ecosistemas (MEA, 2003). El concepto "servicios", integra tanto los beneficios tangibles (provisión) como intangibles (culturales, de regulación y de soporte), los cuales son suministrados por ecosistemas tanto de pequeña como de gran escala (e.g. huertos, milpa, bosques, selvas). Particularmente los SE de provisión tienen la característica de ser finitos, renovables, cuantificables y comercializables (De Groot et al., 2002; Mohri et al., 2013); cuyos aportes van desde la provisión de alimentos, hasta la obtención de materias primas y materiales diversos; en tanto que los servicios intangibles están más relacionados con: la regulación climática, el control de la erosión, el mantenimiento de la calidad del aire, enriquecimiento espiritual, desarrollo cognitivo, y experiencias estéticas (Costanza et al., 1997; MEA, 2005).

Ahora bien, los agroecosistemas de huertos familiares (AEHF) son sistemas tradicionales complejos, los cuales se presentan como alternativa en el suministro de dichos servicios. Dado que son ecosistemas agrícolas conformados por una gran diversidad de especies (generalmente establecidas en una pequeña superficie de tierra) y cuyo manejo se realiza principalmente con los mismos miembros de la familia, resultan ser una de las estrategias imprescindibles en el medio rural (Chávez et al., 2012; Nair, 2001). Dentro de las principales contribuciones de los AEHF están: i) garantizar la seguridad alimentaria, ii) generar ingreso durante la mayor parte del año y iii) permitir la obtención de productos diversos; así como la generación de nuevas redes sociales (Guadarrama et al., 2018; Krishnamurth et al., 2017; Uddin \& Mukul, 2007).

No obstante, estos agroecosistemas de pequeña escala están sufriendo procesos de degradación cada vez más acelerados, relacionados principalmente con la fragmentación de la superficie de la tierra y la pérdida de la agrobiodiversidad (Arcos et al., 2019; Gutiérrez et al., 2017), impactando en la cantidad y calidad de los SE de provisión suministrados, por lo que es necesario crear estrategias para su conservación. En este sentido algunas instituciones han comenzado a revalorar a estos pequeños ecosistemas agrícolas y así se pueden ver diversos estudios entorno a estos, sin embargo, todavía existen vacíos en su abordaje desde una perspectiva ecosistémica e integral. Por lo anterior el objetivo del estudio fue: Realizar un análisis integral de los servicios ecosistémicos de provisión suministrados por agroecosistemas de huertos familiares, en tres comunidades rurales del Estado de México.

\section{MATERIALES Y MÉTODOS}

Área de estudio: El área de estudio se ubica al Sur del Estado de México (1848'58'-

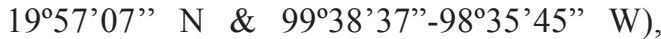
cuenta con una superficie territorial de 614.19 $\mathrm{km}^{2}$ y presenta niveles de altitud, entre los 1 067 m.s.n.m. y 2622 m.s.n.m. (Juan \& Madrigal, 2005). Específicamente se seleccionaron los municipios de Tenancingo, Malinalco y Villa Guerrero; así como una localidad rural de cada municipio, siendo estas: El Carmen, Colonia Juárez y Progreso Hidalgo, respectivamente (Fig. 1). Dado que las localidades se encuentran dentro de una zona de transición ecológica (con condiciones climáticas y la topográficas óptimas), se favorece el desarrollo de una amplia agrobiodiversidad, característica primordial de los huertos familiares (López et al., 2012). El clima es semicálido-templado con lluvias en verano y temperaturas medias anuales que oscilan entre 18.2 y $34.8^{\circ} \mathrm{C}$; y con precipitaciones promedio van de $1305 \mathrm{~mm}$ hasta los $1800 \mathrm{~mm}$ al año. Las rocas presentes son ígneas y sedimentarias, mientras que los tipos del suelo están formados por materiales volcánicos, arcillosos y arenosos. La vegetación está compuesta por bosque mixto de pino-encino en las zonas altas, y selva baja caducifolia en zonas con menor altitud (García, 2004).

\section{Recolecta y análisis de la información:} El estudio se llevó acabo de Marzo a Diciembre 2019, e involucro una revisión de la literatura, en torno a la clasificación de los SE 


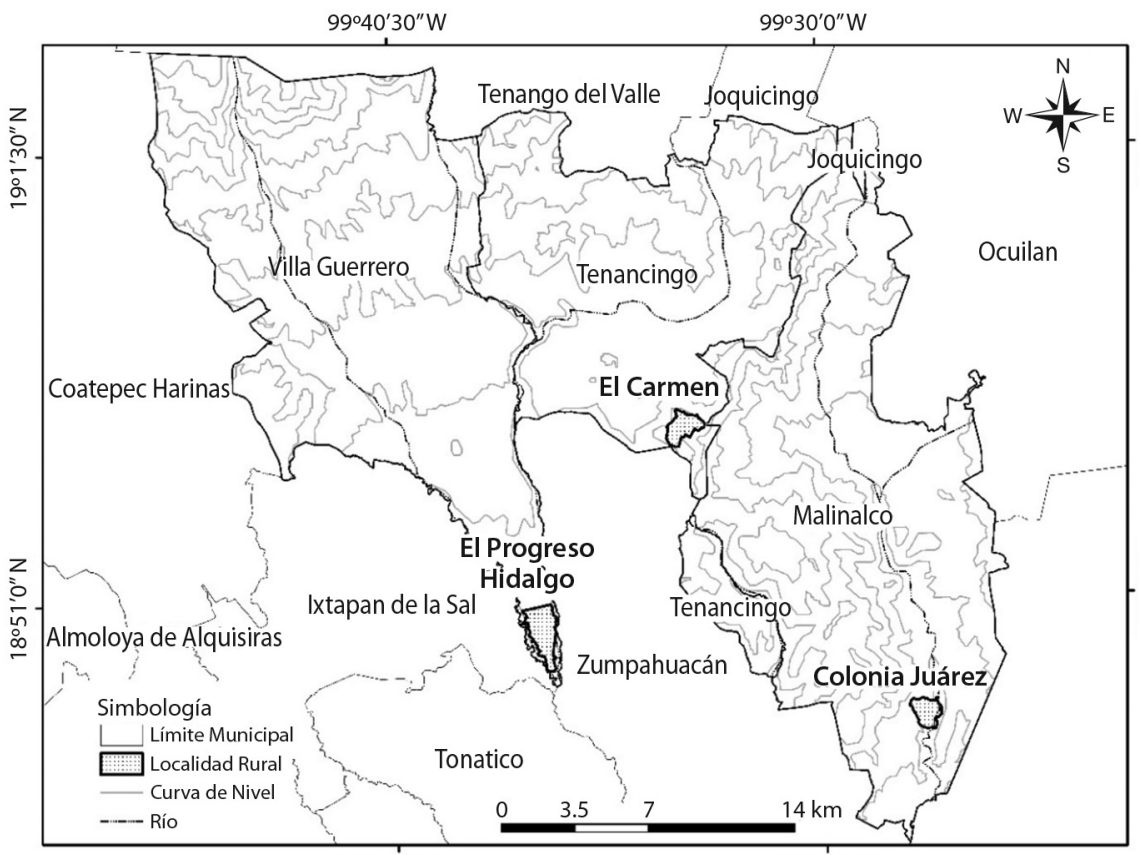

Fig. 1. Municipios y localidades estudiadas. / Fig. 1. Location of the municipalities and localities studied.

ecosistémicos en general y en particular sobre los SE de provisión, de donde se determinó seguir las variantes propuestas por De Groot et al. (2002), Kumar (2010), con su posterior identificación en los huertos familiares seleccionados (Tabla 1). El trabajo empírico, estuvo basado en métodos antropológicos con estancias largas de permanencia en cada una de las comunidades rurales y el uso de cuestionarios, entrevistas semiestructuradas, observaciones directas, recorridos en campo e inventarios de especies. El muestreo estuvo dirigido a 42 propietarios de huertos familiares $(\mathrm{N}=14$ por localidad), quienes fueron seleccionados mediante la técnica conocida como bola de nieve, la cual consistió en ubicar en primera instancia a los informantes clave (hombres y mujeres mayores de 18 años con conocimientos amplios sobre el huerto familiar), estos referenciaron a otros más con las mismas características y así sucesivamente hasta cubrir el tamaño total de la muestra.

La información recolectada, incluyo: (i) la identificación y caracterización de los servicios de provisión (ii) los usos tradicionales de las especies asociadas a los SE de provisión (valor de la importancia de uso), (iii) los destinos de la producción e (iv) información económica relacionada con la generación del ingreso, derivada de la venta de productos. Paralelamente se realizaron inventarios florísticos y faunísticos de las especies que estuvieron asociadas a los servicios de provisión, donde el nombre común de cada especie se recabo con ayuda de los propietarios de huertos, en tanto que la identificación taxonómica, se realizó mediante las guías propuestas por Mariaca (2012), Martínez de la Cruz et al. (2018), Rubí et al. (2014), Villaseñor (2016), White et al. (2013). Dado que la información recolectada era amplia, fue necesario regresar en varias ocasiones a un mismo huerto, lo cual sirvió para verificar mediante observación directa la información que había sido previamente proporcionada por los informantes.

Para determinar la importancia del uso de las especies, se siguió la metodología de la sumatoria de usos modificada por Marín-Corba 
TABLA 1

Servicios ecosistémicos de provisión proporcionados por los huertos familiares de las comunidades rurales del Estado de México

TABLE 1

Provisioning ecosystem services provided by family orchards from rural communities in the State of Mexico

\begin{tabular}{llc}
\hline \multirow{2}{*}{ Categoría de uso } & \multicolumn{1}{c}{ Características de los SE derivados de los AEHF } & Recurso de la identificación \\
\cline { 2 - 3 } Alimenticia & $\begin{array}{l}\text { Variedad de biota vegetal (e.g. frutas, hortalizas, legumbres, granos) y } \\
\text { animal presente en el ecosistema con usos comestibles en fresco. }\end{array}$ & $\bullet$ \\
Condimento & Variedad de biota vegetal utilizada para condimentar los alimentos. \\
Ornamental & Variedad de plantas en el ecosistema con potencial ornamental, cuyo \\
& uso principal es el embellecimiento del hogar. \\
Medicinal & $\begin{array}{l}\text { Variedad de plantas en el ecosistema con potencial medicinal, } \\
\text { empleadas en el tratamiento de enfermedades digestivas, circulatorias, } \\
\text { nerviosas, respiratorias y musculares, entre otras. }\end{array}$ & $\bullet$ \\
Materias primas & $\begin{array}{l}\text { Conversión de la energía solar en biomasa de la cual se obtiene } \\
\text { material para construcción, herramientas agrícolas, abonos vegetales, }\end{array}$ \\
estiércol y forraje. \\
$\begin{array}{l}\text { Industrial y/o } \\
\text { productos } \\
\text { derivados }\end{array}$ & $\begin{array}{l}\text { Diversidad de la biota vegetal y animal utilizada en la obtención de } \\
\text { subproductos alimenticios (e.g. mermeladas, licores, conservas, leche, } \\
\text { huevo, queso, manteca). }\end{array}$ \\
\hline
\end{tabular}

et al. (2005). El cálculo de los ingresos derivados de los servicios de provisión, se realizó considerando: las especies con potencial comercial; la cantidad producida anualmente, las unidades de medida (kilos, pieza o manojo) y los precios de los productos. A partir de esta información fue posible determinar el ingreso bruto anual por especie y los ingresos totales en dólares de los 42 huertos familiares, estos últimos fueron calculados mediante la sumatoria de todas las especies que tuvieron un valor en el mercado. El análisis de la información se realizó de dos maneras: los datos cuantitativos fueron analizados mediante estadística descriptiva (principalmente promedios, porcentajes y frecuencias absolutas), en tanto que los datos cualitativos fueron analizados con base en la información proveniente de la literatura, entrevistas, observaciones y recorridos en campo.

\section{RESULTADOS}

Identificación de los $\mathrm{SE}$ de provisión: Se identificaron un total de seis categorías de servicios ecosistémicos de provisión, de los cuales el $66 \%$ fueron registrados en la literatura (alimenticia, ornamental, medicinal y materias primas), en tanto que el $100 \%$ (alimenticia, condimento, ornamental, medicinal, materias primas e industrial y/o productos derivados) fueron identificados durante las entrevistas, observaciones directas y los recorridos en campo (Tabla 1).

Usos de las especies: Los SE de provisión, estuvieron asociados a 212 especies (202 vegetales y 10 animales), mismas que se distribuyeron en 172 géneros y 82 familias, siendo las más representativas Lamiaceae ( 8 $\%$ ), Asteraceae (7 \%) Fabaceae (6\%), Rosaceae y Crassulaceae (ambas con el $4 \%$ ). Los principales usos que los dueños de los huertos familiares dieron a las especies asociadas fueron: ornamentales (51\%), alimenticios (39 $\%$ ) y medicinales (32\%). A estos le siguieron materias primas $(20 \%)$ y productos derivados $(13 \%)$ en tanto que los usos menos frecuentes fueron como condimento (4\%). El número de especies presente por categoría fue variable, el $2 \%$ estuvo presente en cuatro categorías, el 11 $\%$ en tres, el $31 \%$ en dos, en tanto que el mayor porcentaje $(55 \%)$ se encontró en una. 
Dentro de las especies vegetales con mayor valor de uso estuvieron: Cymbopogon citratus, Persea americana, Punica granatum, Zea mays e Inga jinicuil, con 4 categorías de uso (Tabla 2), en tanto que las especies de Citrus limon, Opuntia streptacantha, Cucurbita ficifolia, Psidium guajava, Musa paradisiaca, Sechium edule y el $50 \%$ de las especies animales principalmente menores (Gallus gallus, Ovis aries, Sus crofa, Capra hircus y Bos indicus), fueron encontradas en 3 categorías de uso. Por el contrario, Foeniculum vulgare, Apium graveolens, Diospyros digyna, Taraxacum officinale, Tanacetum parthenium, Heterotheca inuloides y Ruta chalepensis, fueron de las especies que tuvieron los menores valores de uso (1-2 categorías).

Destino de la producción: Los destinos que los propietarios con AEHF dieron a los productos en fresco fueron: autoconsumo (100 $\%$ de los hogares), venta (50\% del total de los hogares) e intercambio (38\% de los hogares), siendo el primero la principal estrategia de sobrevivencia utilizada por los hogares. En términos generales, el $100 \%$ de los productos tuvieron como principal destino el autoconsumo, el $20 \%$ de estos fueron utilizados adicionalmente con fines de venta, en tanto que el $13 \%$, fueron destinados al intercambio. La Tabla 3, muestra los principales destinos de los principales productos derivados de los SE de provisión, que fueron encontrados con mayor frecuencia en los huertos, de estos se puede destacar sus aportes a la seguridad alimentaria de los hogares, mediante el consumo directo de productos ricos en proteínas (derivados de aves, carne de res, cerdo y leguminosas), vitaminas (A, E, C), minerales como hierro, potasio, magnesio y calcio (presentes en las hojas de los vegetales) y carbohidratos (derivado de las diferentes frutas y gramíneas).

Por otro lado, la venta, representó la forma más usual en la obtención del ingreso, donde los hogares que vendieron utilizaron el $66 \%$ de la producción para estos fines y el restante $34 \%$ fue utilizada para el consumo familiar, lo cual significo un ahorro de 686.93 USD/año al

TABLA 2

Especies asociadas a los servicios ecosistémicos de provisión de AEHF con mayor valor de uso

TABLE 2

Species associated with provisioning ecosystem services of AEFO with higher use value

\begin{tabular}{llccc}
\hline \multicolumn{1}{c}{ Especies } & \multicolumn{1}{c}{ Familia } & Frecuencia (N=42) & Usos & Sumatoria de usos \\
\hline Cymbopogon citratus (DC.) Staff. & Poaceae & 20 & $1,3,4,5$ & 4 \\
Persea americana Mill. & Lauraceae & 18 & $1,3,4,6$ & 4 \\
Punica granatum L. & Punicaceae & 17 & $1,3,4,6$ & 4 \\
Zea mays L. & Poaceae & 7 & $1,3,4,5$ & 4 \\
Inga jinicuil Schltr. \& Cham. ex G. Don. & Fabaceae & 1 & & 4 \\
Citrus limon (L.) Osbeck & Rutaceae & 27 & $1,3,4$ & 3 \\
Opuntia streptacantha Lem. & Cactaceae & 22 & $1,4,6$ & 3 \\
Cucurbita ficifolia Bouché & Cucurbitaceae & 18 & $1,5,6$ & 3 \\
Psidium guajava L. & Myrtaceae & 17 & $1,4,6$ & 3 \\
Musa paradisiaca L. & Musaceae & 12 & $1,3,4$ & 3 \\
Sechium edule (Jacq.) Sw. & Cucurbitaceae & 12 & $1,4,6$ & 3 \\
Carica papaya L. & Caricaceae & 8 & $1,3,4$ & 3 \\
Juglans regia L. & Juglandaceae & 8 & $1,3,4$ & 3 \\
Justicia spicigera Schltdl. & Acanthaceae & 8 & $3,4,5$ & 3 \\
Prunus serotina ssp capuli (Cav.) Mac Vaugh & Rosaceae & 6 & $1,4,6$ & 3 \\
Rubus caudatisepalus Calderón & Rosaceae & 6 & $1,4,6$ & 3 \\
\hline
\end{tabular}

Usos documentados: 1 = Alimenticio; 2 = Condimento; 3 = Ornamental; 4 = Medicinal; $5=$ Materias primas; $6=$ Industrial y/o productos derivados. 
TABLA 3

Frecuencia y destino de los productos encontrados con mayor frecuencia en los huertos familiares del Estado de México

TABLE 3

Frequency and destination of provisioning ecosystem services in 42 family orchards from State of Mexico

\begin{tabular}{lcccc}
\hline \multicolumn{1}{c}{ Especies } & Frecuencia $(\mathrm{N}=42)$ & Autoconsumo \% & Venta $\%$ & Intercambio \% \\
\hline Chenopodium ambrosioides L. & 31 & 100 & 0 & 0 \\
Mentha piperita L. & 30 & 100 & 0 & 0 \\
Citrus limon (L.) Osbeck & 27 & 77 & 23 & 0 \\
Rosa indica L. & 27 & 90 & 0 & 10 \\
Gallus gallus & 25 & 85 & 15 & 0 \\
Aloe barbadensis Mill. & 24 & 90 & 0 & 10 \\
Opuntia streptacantha Lem & 22 & 75 & 35 & 0 \\
Cymbopogon citratus (DC.) Staf & 20 & 100 & 0 & 0 \\
Tanacetum parthenium (L.) Sch. Bip. & 19 & 10 & 0 & 0 \\
Persea americana Mill. & 18 & 10 & 83 & 7 \\
Psidium guajava L. & 17 & 25 & 85 & 5 \\
Prunus persica (L.) Batsch. & 14 & 100 & 73 & 5 \\
Capsicum pubescens Ruiz \& Pav. & 12 & 100 & 0 & 2 \\
Spathodea campanulata P. Beauv. & 12 & & 0 & 0 \\
Agave atrovirens Karw. Ex Salm-Dyck & 11 & & & 0 \\
\hline
\end{tabular}

evitar la compra de productos, en tanto que los hogares que no vendieron tuvieron un ahorro de 1222.79 USD/año. Los ingresos fueron utilizados principalmente en la compra de otros productos alimenticios (e.g. azúcar, sal, pastas, granos, cereales y pan) contribuyendo de esta manera con la dieta alimenticia de los hogares. Finalmente, y aunque en menor medida el intercambio facilito entre otras cosas: la obtención de otros productos, la generación de nuevo material genético (ornamental) y el establecimiento de nuevas relaciones sociales, como efecto de la interacción entre los propietarios de los huertos, compradores y otros vendedores.

Generación del ingreso: Los productos comestibles fueron los principales aportadores del ingreso. Dentro de las especies más con mayor potencial comercial estuvieron: Persea americana, Prunus persica, Citrus limon, Annona cherimola, Annona muricata, Manilkara sapota, Pouteria sapota, Mangifera indica, Punica granatum, Passiflora edulis, Capsicum pubescens, Vicia faba, Sechium edule, Bos indicus y Gallus gallus. A partir de estas especies se obtuvieron adicionalmente diversos productos derivados, cuya elaboración implico el uso de aquellas que tuvieron un alto grado de maduración o que fueron de difícil colocación en el mercado como productos en fresco, no obstante que la venta de estos represento un mayor valor económico en comparación con su venta en fresco.

Los productos obtenidos, fueron comercializados en mercados locales y municipales (cercanos a las comunidades), cuya venta directa genero ingresos brutos anuales de 1333.47 USD en promedio. Este valor presento variabilidad ( $\mathrm{SD}=930.04)$, dado que se encontraron huertos con valoraciones económicas muy bajas (501.68 USD/año) y otros con valoraciones de hasta 3630.60 USD/año. En este sentido, los ingresos fueron obtenidos principalmente de productos frutales (878.65 USD/año), seguida de la venta de animales (287.08 USD/año) y productos derivados (110.68 USD) (Tabla 4). De estos últimos se pueden destacar como más vendidos los productos de origen animal como huevo (91 kg/año), queso (70 piezas/año) y leche (50 L/año) y en menor medida los de origen vegetal como conservas (48 pzas/año), 
TABLA 4

Ingresos brutos derivado de los servicios ecosistémicos de provisión de AEHF, en dólares

TABLE 4

Gross income derived of provision ecosystem services from AEFO, in dollars

\begin{tabular}{llcc}
\hline \multicolumn{1}{c}{ Categoría de uso } & \multicolumn{1}{c}{ Productos } & Frecuencia $(\mathrm{N}=21)$ & Ingresos totales $( \pm \mathrm{SD})$ \\
\hline Alimenticia & Frutales & 19 & $878.65( \pm 881.83)$ \\
& Animales domesticados & 7 & $287.08( \pm 510.73)$ \\
& Hortalizas & 6 & $56.51( \pm 113.72)$ \\
Productos derivados & Vegetales y animales & 11 & $110.68( \pm 237.38)$ \\
& & & $1333.47( \pm 930.04)$ \\
\hline
\end{tabular}

licores (30 pzas/año), cristalizados (25 pzas/ año), almíbar (20 pzas/año), mermeladas (12 pzas/año) y aceites (10 pzas/año).

\section{DISCUSIÓN}

El presente estudio se enfocó en el análisis integral de los servicios ecosistémicos de provisión proporcionados por los huertos familiares, con la incorporación de características biofísicas y económicas, lo cual es difícil de observar en la mayoría de los estudios enfocados en agroecosistemas de pequeña escala (Kumar \& Nair, 2006). Ahora bien, el análisis partió de la identificación y clasificación de los SE de provisión de acuerdo al uso principal de las especies. De esta manera se determinaron seis categorías de uso, las cuales estuvieron claramente diferenciables y no se traslaparon entre sí, situación que permitió realizar un mejor análisis de los servicios encontrados. En este sentido se plantea que la categorización aquí propuesta podría servir de modelo para el abordaje de los servicios de provisión de los huertos familiares de otras regiones; así como de otros agroecosistemas y al mismo tiempo realizar comparaciones con base en una misma clasificatoria.

La diversidad asociada a los servicios de provisión registrada aquí fue de 212 especies, mismas que se distribuyeron en 172 géneros y 82 familias, no obstante que las especies vegetales (202 spp.) predominaron sobre la fauna domesticada (10 spp.), situación que estuvo en concordancia con lo reportado por otros autores en estudios sobre huertos familiares de México. Mariaca (2012), al estudiar varias regiones del país encontró que la diversidad vegetal fue superior (entre 150 y 301 spp. vegetales), a la faunística (17 spp.); Chablé et al. (2015), encontraron $330 \mathrm{spp}$. de vegetales y $17 \mathrm{spp}$. de fauna domesticada en huertos de Tabasco; Castañeda et al. (2020), documentaron 357 spp. vegetales (distribuidas en 263 géneros y 102 familias botánicas) y $20 \mathrm{spp}$. animales en huertos de Puebla México.

Los principales usos que los propietarios de los huertos dieron a las especies asociadas fueron: ornamentales (51\%), seguidos de los alimenticios (39\%) y medicinales (32\%). Resultados similares fueron encontrados por Blanckaert et al. (2004), Moctezuma (2014), Bautista et al. (2016). Por el contrario, Guarneros et al. (2014), Chablé et al. (2015), identificaron como principal uso el alimenticio, en tanto que Rebollar et al. (2008), White et al. (2017), mencionaron a la categoría medicinal como la más ampliamente utilizada. Estas diferencias de uso muy probablemente se deban a las preferencias, gustos y necesidades que los dueños de los huertos familiares presentan al momento de decidir que especies establecer, aunado a las características de cada lugar, lo que finalmente determina el uso final de las especies.

El destino de la producción fue: autoconsumo, venta e intercambio, siendo el primero la principal estrategia de sobrevivencia utilizada por los hogares. Los productos comestibles fueron los principales aportadores de alimentos nutritivos, generación del ahorro e ingresos 
(1 333.47 USD/año). Aunque los ingresos fueron similares a los reportados por Kumari et al. (2009), otros autores como Reyes-García et al. (2012) registraron ingresos superiores en huertos de España, donde las especies hortícolas fueron las principales aportadoras. Estas variaciones en los ingresos muy probablemente están determinadas por la superficie del terreno, productos ofertados y los precios en el mercado. Adicionalmente pueden estar asociados, diversos factores económicos, principalmente aquellos relacionados con los apoyos con los que cuentan los agricultores de países desarrollados, a diferencia de los agricultores de los países en vías de desarrollo.

Declaración de ética: los autores declaran que todos están de acuerdo con esta publicación y que han hecho aportes que justifican su autoría; que no hay conflicto de interés de ningún tipo; y que han cumplido con todos los requisitos y procedimientos éticos y legales pertinentes. Todas las fuentes de financiamiento se detallan plena y claramente en la sección de agradecimientos. El respectivo documento legal firmado se encuentra en los archivos de la revista.

\section{AGRADECIMIENTOS}

Agradecemos al Consejo Nacional de Ciencia y Tecnología (CONACYT), por el apoyo recibido en el financiamiento de la investigación. Se reconoce el apoyo otorgado por parte de las autoridades locales para facilitarnos el acceso a las comunidades y aproximarnos a los locatarios. De manera especial se agradece a los hogares de las diferentes comunidades rurales por su valiosa participación y colaboración en el presente proyecto, sin los cuales hubiera sido imposible realizar el trabajo de campo.

\section{RESUMEN}

Introducción: Los servicios ecosistémicos (SE) de provisión suministrados por los agroecosistemas de huertos familiares (AEHF) se refieren a los beneficios tangibles que las personas obtienen del ecosistema. Aunque los AEHF, han sido ampliamente estudiados, pocos son los trabajos que evidencian su abordaje desde una perspectiva integral, la cual incorpore características biofísicas y económicas al mismo tiempo.

Objetivo: Realizar un análisis integral los servicios ecosistémicos de provisión suministrados por agroecosistemas de huertos familiares del Estado de México.

Métodos: Se recopilo información sobre los SE de provisión (identificación, usos, destinos y generación de ingresos) mediante entrevistas semiestructuradas, cuestionarios, inventarios de especies, observaciónes directas, y recorridos en campo entre Marzo a Diciembre de 2019 en tres comunidades rurales, con la participación de 42 familias propietarias de los huertos familiares.

Resultados: Se identificaron un total de seis SE de provisión asociados a 212 especies, las cuales fueron distribuidas en 172 géneros y 82 familias. Los Principales usos que los propietarios de los huertos dieron a los SE de provisión fueron ornamentales $(51 \%)$, seguidos de los alimenticios (39\%) y medicinales (32\%), donde especies como Cymbopogon citratus, Persea americana, Punica granatum, Zea mays e Inga jinicuil, tuvieron los mayores valores de uso (V. U. =4). El destino de la producción fue autoconsumo, venta e intercambio; siendo el primero, la principal estrategia de sobrevivencia utilizada por los hogares. La venta de productos comestibles en fresco, así como de sus derivados represento ingresos brutos promedio de 1333.47 USD/año para los propietarios de los huertos familiares.

Conclusiones: Los servicios ecosistémicos de provisión proporcionados por los huertos familiares son vistos como una fuente importante de recursos, los cuales permiten satisfacer necesidades múltiples de los hogares rurales, catalogados en pobreza extrema.

Palabras clave: agrobiodiversidad; valoración económica; agroecosistemas tropicales; análisis interdisciplinario, conocimiento local.

\section{REFERENCIAS}

Arcos, M., Gutiérrez, J. G., \& Balderas, M. A. (2019). Diagnóstico de huertos familiares, en tres localidades rurales al sur del Estado de México. En A. Huerta., F. García., L. A. Villarreal, \& J. A. Salazar (Eds.), Agricultura Sostenible. Por la tierra por la vida (pp. 431-435). Universidad Autónoma Chapingo, México.

Bautista, G. Á., Sol, A., Velázquez, A., \& Llanderal, T. (2016). Composición florística e importancia socioeconómica de los huertos familiares del Ejido La Encrucijada, Cárdenas, Tabasco. Revista Mexicana de Ciencias Agrícolas, 14, 2725-2740.

Blanckaert, I., Swennen, R. L., Paredes, M., \& Saade, R. L. (2004). Floristic composition, plant uses and management practices in homegardens of San Rafael 
Coxcatlan, Valley of Tehuacan-Cuicatlan, México. Journal of Arid Environments, 57, 39-62. https://doi. org/10.1016/S0140-1963(03)00100-9

Castañeda, I., Aliphat, M., Caso, L., Lira, R., \& Martínez, D. (2020). Conocimiento tradicional y composición de los huertos familiares totonacas de Caxhuacan, Puebla, México. Polibotánica, 49, 185-217. https:// doi.org/10.18387/polibotanica.49.13

Chablé, R., Palma, D. J., Vázquez, C. J., Ruiz, O., Mariaca, R., \& Ascensio, J. M. (2015). Estructura, diversidad y uso de las especies en huertos familiares de la Chontalpa, Tabasco, México. Ecosistemas y Recursos Agropecuarios, 2(4), 23-39.

Chávez, E., Rist, S., \& Galmiche, Á. (2012). Lógica de manejo del huerto familiar en el contexto del impacto modernizador en Tabasco, México. Cuadernos de Desarrollo Rural, 9(68), 177-200.

Costanza, R., D’Arge, R., De Groot, R. S, Farber, S., Grasso, M., Hannon, B., Limburg, K., Naeem, S., O’Neill, R. V., Paruelo, J., Raskin, R. G., Sutton, P., \& Van den Belt, M. (1997). The value of the world's ecosystem services and natural capital. Nature, 387(1), 253-60. https://doi.org/10.1038/387253a0

De Groot, R. S., Wilson, M. A., \& Boumans, R. M. J. (2002). A typology for the classification, description and valuation of ecosystem functions, goods and services. Ecological Economics, 41, 393-408. https://doi.org/https://doi.org/10.1016/S0921-8009 (02)00089-7

García, E. (2004). Modificaciones al Sistema de Clasificación Climática de Köppen. Editorial Universidad Nacional Autónoma de México, México.

Guadarrama, N., Sangerman-Jarquín, D., Chávez, C., \& Rubí, M. (2018). Estrategias de comercialización de los frutos en el tianguis de Malinalco, México. Revista Mexicana de Ciencias Agrícolas, 9(4), 841-849. https://doi.org/10.29312/remexca.v9i4.1400

Guarneros, N., Morales, J., Cruz, J., Huerta, A., \& Ávalos, A. D. (2014). Economía familiar e índice de biodiversidad de especies en los traspatios comunitarios de Santa María Nepopualco, Puebla. Revista Mexicana de Ciencias Agrícolas, 9, 1701-12.

Gutiérrez, J. G., García, J. C., \& Franco, R. (2017). La tradición agroecológica de los huertos familiares. La importancia de su conservación. En R. Jarquín \& A. Huerta (Eds.), Agricultura sostenible como base para los agronegocios (pp. 407-417). Universidad Autónoma de San Luis Potosí, México.

Juán, J. I., \& Madrigal, D. (2005). Huertos, diversidad y alimentación en una zona de transición ecológica del Estado de México. Ciencia Ergo Sum, 12, 54-63. https://doi.orgredalyc.org/pdf/104/10412106.pdf
Krishnamurth, L. R., Krishnamurthy, S., Rajagopal, I., \& Peralta, A. (2017). Agricultura familiar para el desarrollo rural incluyente. Terra Latinoamericana, $35,135-47$.

Kumar, B. M., \& Nair, P. K. R. (2006). Tropical Homegardens. Advances in Agroforestry. Springer. https://doi. org/10.1007/978-1-4020-4948-4

Kumar, P. (2010). The Economics of Ecosystems and Biodiversity. Ecological and Economic Foundations. Routledge.

Kumari, M. A., Kansuntisukmongkol, K., \& Brockelman, W. Y. (2009). Plant Diversity in Home Gardens and Its Contribution to Household Economy in Suburban Areas in Sri Lanka. Environment and Natural Resources Journal, 7(2), 12-30. https://ph02.tci-thaijo.org/ index.php/ennrj/article/view/82565

López, E. J., López, J. A., Beltrán, A. S., \& Aguilera, L. I. (2012). Composición de la flora arbórea en el área natural protegida Tenancingo Malinalco-Zumpahuacán, Estado de México, México. Polibotánica, 34, 51-98. http://www.scielo.org.mx/pdf/polib/n34/ $\mathrm{n} 34 \mathrm{a} 3$

Mariaca, M. R. (2012). La complejidad del huerto familiar Maya del sureste de México. En M. R Mariaca (Ed.), El huerto familiar del sureste de México (pp. 7-97). Colegio de la Frontera Sur, México.

Marín-Corba, C., Cárdenas-López, D., \& Suárez-Suárez, S. (2005). Utilidad del valor de uso en etnobotánica. Estudio en el departamento de Putumayo (Colombia). Caldasia, 27(1), 89-101.

Martínez de la Cruz, I., Villaseñor, J. L., Aguilera Gómez, L. I., \& Rubí Arriaga, M. (2018). Angiospermas nativas documentadas en la literatura para el Estado de México, México. Acta Botánica Mexicana, 124, 135217. https://doi.org/10.21829/abm124.2018.1273

Millennium Ecosystem Assessment (MEA) (2005). A Report of the Millennium Ecosystem Assessment. Ecosystems and Human Well-Being. Island Press.

Millennium Ecosystem Assessment (MEA). (2003). Ecosystems and Human Well-Being: A Framework for Assessment. Island Press.

Moctezuma, S. (2014). Cambios en la biodiversidad de los huertos familiares en una comunidad del suroeste de Tlaxcala. Sociedad y Ambiente, 1(4), 4-22. https:// doi.org/10.31840/sya.v0i4.1521

Mohri, H., Lahoti, S., Saito, O., Mahalingam, A., Gunatilleke, N., Van Thang Hoang, I., Hitinayake, G., Takeuchi, K., \& Herath, S. (2013). Assessment of ecosystem services in Homegarden systems in Indonesia, Sri Lanka, and Vietnam. Ecosystem Services, 5, 124-36. http://dx.doi.org/10.1016/j. ecoser.2013.07.006 
Nair, P. K. R. (2001). Do tropical homegardens elude science, or is it the other way around? Agroforestry Systems, 53(2), 239-45. https://doi. org/10.1023/A:1013388806993

Rebollar, S., Santos, V. J., Tapia, N. A., \& Pérez, C. P. (2008). Huertos familiares, una experiencia en Chancah Veracruz, Quintana Roo. Polibotánica, 25, $135-154$

Reyes-García, V., Aceituno, L., Vila, S., Calvet-Mir, L., Garnatje, T., Jesch, A., Lastra, J. J., Parada, M., Rigat, M., Valles, J., \& Pardo-de-Santayana, M. (2012). Home gardens in three mountain regions of the Iberian Peninsula: description, motivation for gardening, and gross financial benefits. Journal of Sustainable Agriculture, 36, 249-270. http://dx.doi.org/10.1080/ 10440046.2011.627987

Rubí, M., Martínez, I., González, A; Pérez, D., Cruz, J., \& Guadarrama, N. (2014). Catálogo de especies frutales presentes en el sureste del Estado de México, México. Revista Mexicana Ciencias Agrícolas, 5(8), 1509-1517.
Uddin, M. B., \& Mukul, S. A. (2007). Improving Forest Dependent Livelihoods Through NTFPs and Home Gardens: A Case Study from Satchari National Park Mohammad. In J. Fox, B. R. Bushley, S. Dutt, \& A. Q. Shimona (Eds.), Making Conservation Work: Linking Rural Livelihoods and Protected Areas in Bangladesh (pp.13-35). East-West Center, Honolulu and Nishorgo Program of the Bangladesh Forest Department, Dhaka.

Villaseñor, J. L. (2016). Checklist of the native vascular plants of Mexico. Catálogo de las plantas vasculares nativas de México. Revista Mexicana de Biodiversidad, 87(3), 559-902.http://dx.doi.org/10.1016/j. rmb.2016.06.017

White, L., Juan, J. I., Chávez, C., \& Gutiérrez, J. G. (2013). Flora medicinal en San Nicolás, municipio de Malinalco, Estado de México. Polibotánica, 35, 173-206.

White, L., Chávez, C., \& García, D. (2017). Análisis del estrato arbóreo de agroecosistemas en una zona de transición ecológica. Ecosistemas y Recursos Agropecuarios, 4(11), 255-64. https://doi.org/10.19136/ era.a4n11.882 